\title{
A systematic review and meta-analysis on the effect of reminiscence therapy for people with dementia
}

\author{
Kyongok Park, ${ }^{1}$ Seonhye Lee, ${ }^{2}$ JeongEun Yang, ${ }^{3}$ Taekwon Song, ${ }^{3}$ \\ and Gwi-Ryung Son Hong ${ }^{3}$ \\ ${ }^{1}$ Department of Nursing, Gangneung-Wonju National University, Wonju, Republic of Korea \\ ${ }^{2}$ Department of Nursing, Gyeongnam National University of Science and Technology, Finju-si, Republic of Korea \\ ${ }^{3}$ College of Nursing, Hanyang University, Seoul, Republic of Korea
}

ABSTRACT

Background: The main objective of this study was to conduct a meta-analysis to identify the effects of reminiscence therapy in people with dementia (PWD).

Methods: A systematic search of randomized controlled trials (RCTs) was conducted using bibliographic databases.
A total of 157 original published studies were identified in the search, and 24 complete articles were included in the
final review to check for the level of evidence. Two of the study authors independently assessed the quality of the
included studies using the "Risk ofBias" (ROB) tool developed by the Cochrane Collaboration. Depression, quality
of life, and behavioral and psychological symptoms of dementia (BPSD) were selected to measure the effect of
reminiscence therapy. To determine the effects ofreminiscence therapy on these variables, each individual studywas
analyzed using Comprehensive Meta-Analysis Software ${ }^{\circledR}$ (Biostat, Englewood, NJ, USA).

Results: The overall effect size was presented using standardized mean differences (SMDs) and 95\% confidence intervals. Cohen's $d$ effect size for depression was -0.541 (95\% CI: -0.847 to $-0.234, Z=-3.730, p<0.001$ ), indicating that depression was significantly reduced in the reminiscence group compared to the control group. Increased quality of life and decreased BPSD were also found in the reminiscence group compared to the control group.

Conclusion: Reminiscence therapy has a moderate effect on depression and can be broadly used to decrease depression as an alternative to antipsychotics, which can have harmful side effects and high cost.

Key words: reminiscence, dementia, randomized controlled trial, depression, quality of life, cognition

\section{Introduction}

The number of patients with dementia increased to $35,600,000$ in 2010 and is expected to double every 20 years, estimating that the number of dementia patients will be 65,700,000 in 2030 and $115,400,000$ in 2050 (WHO, 2012). To reduce the behavioral and psychological symptoms of dementia (BPSD), various pharmacological and non-pharmacological treatments have been used for people with dementia (PWD). Some studies have reported that there was no effect of pharmacological interventions on the BPSD (Gitlin et al., 2009), while a few studies found

Correspondence should be addressed to: Gwi-Ryung Son Hong, College of Nursing, Hanyang University, \#222 Wangsimni-ro, Sungdong-gu, Seoul 04763, Korea. Phone: (+82-2) 2220-0701; Fax: (+82-2) 2295-2074. Email: grson@hanyang.ac.kr. Received 17 Oct 2017; revision requested 21 Nov 2017; revised version received 06 Nov 2018; accepted 10 Nov 2018. First published online 04 February 2019. potential benefits to using non-pharmacological interventions for depression (Duru Aşiret and Kapucu, 2016), on cognitive function (Carrion et al., 2013), and social activity (Akanuma et al., 2011).

Reminiscence therapy was developed by Butler as a treatment for dementia using a process of "Life Review" (Butler, 1963) in which an individual recalls their past events, activities, and experience to help give meaning to their lives. The life review process uses various memory triggers such as household items, objects from the past, photographs, and music (Woods et al., 2012). Reminiscence therapy has been used broadly as a non-pharmacological intervention and can be conducted with a group or individually (Woods et al., 2005). Generally, groupbased reminiscence therapy is commonly used since it allows participants to stimulate each other through conversation and increases their attention span. 
Individual reminiscence therapy also has benefits due to its focus on a person-centered approach.

Dementia describes a collection of symptoms that are caused by disorders affecting the brain. Since it was introduced in the early 1960s for patients with psychological signs and symptoms or in the frail elderly (Buchanan and Middleton, 1994), reminiscence therapy has been reported to be effective in reducing depression (Duru Assiret and Kapucu, 2016) for PWD. Reminiscence therapy could allow PWD to better manage BPSD (Tadaka and Kanagawa, 2007) and improve (O'Shea et al., 2014) and slightly benefit on their quality of life (QoL) (Woods, et al., 2018), which can contribute to reduce the care burden on caregivers. In terms of QoL, there are only available measurements for participants with relatively high cognitive function, indicating a limitation to identify the effectiveness of reminiscence therapy.

Although BPSD as an effective outcome variable with reminiscence therapy is controversial by previous literature, BPSD is worthwhile to be examined because it is the factor causing the most stress on formal and informal caregivers of PWD. In addition, current scientific evidence is not sufficiently available for the occurrence and management of BPSD. Many researchers are trying to decrease the BPSD in PWDs by utilizing many different non-pharmacological interventions. Therefore, it must be valuable to examine the effect of reminiscence therapy on BPSD. Moreover, some other studies failed to prove the effectiveness of this therapy with regard to depression (Wang, 2007), BPSD (Baines et al., 1987), and QoL (Lai et al., 2004) in PWDs. Therefore, it is uncertain whether reminiscence therapy has an effect in PWDs or what symptoms might be affected.

Because of this knowledge gap, a systematic review and meta-analysis of the published literature were performed to identify the overall effects of reminiscence therapy for PWD. A recent systematic review also reported that evidence of the therapeutic effect was not robust (Subramaniam and Woods, 2012; Woods et al., 2012; 2018) because of the small number of studies. Huang et al. (2015) reported in their systematic review and meta-analysis that reminiscence therapy had an effect on depressive symptoms in PWDs, and they also tried to demonstrate the long-term effect of reminiscence therapy, although the study (Huang et al., 2015) only examined depressive symptoms. Unlike pharmacological intervention, which tends to target only one symptom, we examined the effects of reminiscence therapy on various outcomes for PWDs including depression, QoL, and BPSD. We included a large number of RCTs to produce a robust result.

Regardless of this argument, reminiscence therapy has been known that there is practical advantages since it is a simple intervention with low risk for application to PWD (Haight et al., 2006; van Bogaert et al., 2013). Non-pharmacological interventions that can slow disease progression are currently becoming increasingly important as an addition to pharmacological treatments (Ngo and Holroyd-Leduc, 2014)

Therefore, this meta-analysis will identify the effects of reminiscence therapy on depression, quality of life, and BPSD among PWDs. In addition, this meta-analysis will identify the evidence as to whether individual or group reminiscence therapy can have better therapeutic effect, and what frequency of interventional sessions is suitable to obtain a therapeutic effect through the subgroup analysis.

\section{Methods}

A systematic search for randomized controlled trials (RCTs) was conducted from July to August 2016. The bibliographic databases CENTRAL, CDSR, DARE, MEDLINE, CINAHL, and ProQuest and the Korean databases RISS, KISS, and NDSL were used for the systematic search of RCTs. The keywords used in the search were as follows: ("reminiscence" OR "reminiscence therapy" OR "reminiscence intervention" OR "reminiscence treatment" OR "reminiscence program" OR "spiritual reminiscence" OR "structured reminiscence therapy" OR "memory reminiscence" OR "life review" OR "life story book") AND ("randomized controlled trial") AND ("dementia" OR "Alzheimer's" OR "Alzheimer's disease" OR "Alzheimer's dementia" OR "vascular dementia"). Manual searching was also performed to include articles that might have been omitted from the electronic search.

A total of 157 original published studies were identified in the search, and 121 studies remained after deleting duplicates. Two authors independently reviewed the titles and abstracts of the studies and excluded studies that did not include reminiscence therapy, an RCT research design, and PWDs. Finally, 24 complete articles were included in the review to check for the level of evidence. The flow diagram of the study screening is shown in Figure 1. Two authors reviewed all of the papers to determine if the studies fit the inclusion criteria. When the two authors disagreed during the study evaluation, disagreement was resolved by discussion and/or consultation with a third author.

\section{Inclusion criteria}

Only studies using randomized controlled trials as the primary study design were included; however, while reviewing the studies, we found that it was difficult to evaluate the blindness and randomization 


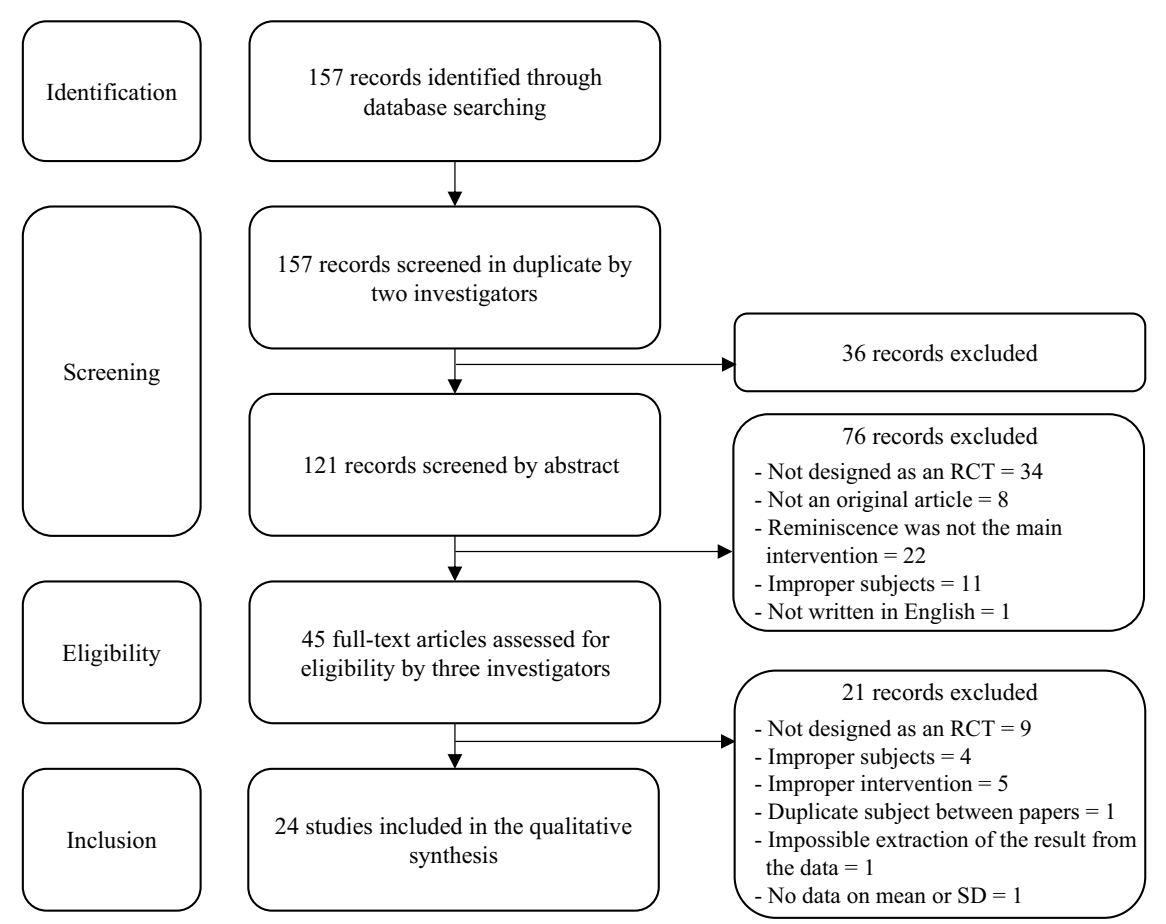

Figure 1. Flow diagram of the study screening.

of studies on non-pharmacological interventions. There were unclear explanations on "random sequence generation" or "allocation concealment" when reviewing the studies; therefore, we included studies if the RCTs were applied using either "random sequence generation," "allocation concealment," or both based on the recommendation of CONSORT (Consolidated Standards of Reporting Trials) (Moher et al., 2001). If there was only a statement on applying random assignment without any explanation of the randomization or detailed randomization method, we categorized the potential study into the 'unclear bias' section of randomization.

The inclusion criteria for the participants were age over 60 years, diagnosis of dementia such as Alzheimer's disease or vascular dementia, and cognitive decline. We mainly selected studies including participants with mild to moderate dementia. The severity of dementia was mostly evaluated by the Mini-Mental State Examination (MMSE) or Clinical Dementia Rating (CDR), and participants with an MMSE score between 10 and 24 or CDR score of 1-2 were selected for this study. If there were both dementia and non-dementia participants in a study, we only included the results from the patients diagnosed with dementia.

Studies using reminiscence therapy in which demented elderly participants recalled the past in groups or individually were included in the analysis. This therapy also uses aides such as photographs, music, and videos from the past. Studies with a minimum study period of four weeks were included.
If the intervention used other therapies such as exercise, printing, music, gardening, or usual care, we only included the results from the reminiscence therapy. Interventions that did not influence the main variables such as no treatment, usual care, physical activity, or informal social contact were included in the control group (Akanuma et al., 2011; Azcurra, 2012; Kwon et al., 2012; Nakatsuka et al., 2015). If studies had more than three groups such as an intervention, comparison, and control group, we analyzed the results from the reminiscence group and control group; however, if we were not able to extract the results from the reminiscence or control group, the study was excluded.

The outcome variables considered for the inclusion criteria were depression, QoL, and BPSD as primary outcomes to measure the effect of reminiscence therapy. Depression was typically measured by the self-reported Geriatric Depression Scale (GDS) and Cornell Scale for Depression in Dementia (CSDD) as an observed scale. Most studies assessed QoL with the Quality of Life in Alzheimer's Disease (QoL-AD) measurement. BPSDs were measured by various instruments such as the Behavioral Rating Scale (BRS), Clifton Assessment Procedures for the Elderly (CAPE), and CohenMansfield Agitation Inventory (CMAI).

\section{Exclusion criteria}

Exclusion criteria of this study were as follows: 1) studies including participants who were diagnosed with psychiatric disorders such as depression, 2) 


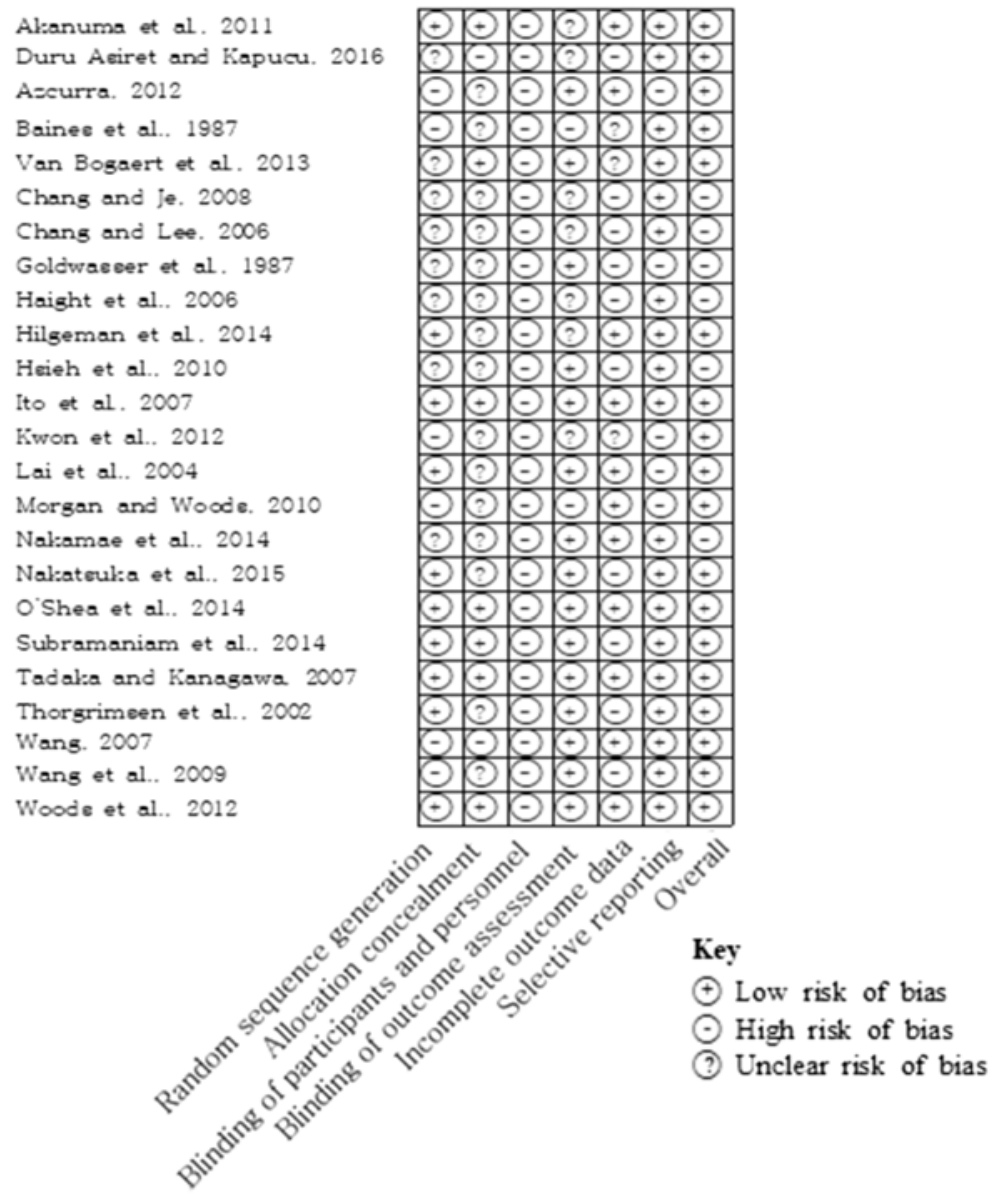

Figure 2. The Cochrane Collaboration tool for assessing risk of bias.

studies that did not extract results on the effect of reminiscence therapy including reality orientation or multi-sensory treatment, 3) qualitative or case control studies, 4) protocol and/or pilot studies that did not report results on the effect of reminiscence therapy, and 5) studies that were not written in English or Korean.

\section{Assessment of the risk of bias}

Evaluating the risk of bias (ROB) is one of the key steps in a systematic review and assessment the internal validity of the study, and eventually, evaluating ROB allows one to assess the strength of evidence (Hartling et al., 2012). Therefore, ROB was evaluated as a first step of a systematic review. However, this approach can only be used to interpret the quality of studies, not to merge the results as part of a quantitative evaluation. Therefore, we conducted a meta-analysis as the next step to identify the effect of reminiscence therapy on the selected variables.

Two of the study authors independently assessed the quality of the included studies using the "Risk of Bias (ROB)" tool developed by the Cochrane Collaboration, which is illustrated in Figure 2 (Higgins et al., 2011). The Risk of Bias tool assesses random sequence generation, allocation concealment, blinding of participants and personnel, blinding of outcome assessment, incomplete outcome data, selective outcome reporting, and other sources of bias. We classified the ROB of the studies as "low risk," "high risk," or "unclear risk."

Eighteen out of 24 RCTs were evaluated as having low risk of bias, which indicates a high quality study, and 6 RCTs were evaluated as having high risk of bias. When the random sequence generation method was assessed to evaluate the selective bias, 10 RCTs were evaluated as low risk of bias because they conducted a simple randomization using a computer or stratified randomization considering age, level of education, type of dementia, or type of institution. Six RCTs were evaluated as having high risk of bias. For example, if subjects with even numbers were assigned to the experimental group, the study was evaluated as having a high risk of bias because the odd or even numbers of a table list are a systematic or non-random approach compared to sequence generation by computer random number generation or coin tossing (Higgins et al., 2011). Studies without descriptions of selection bias were evaluated as unclear bias. 
Blinding was evaluated based on whether the study participants and key study personnel were blinded to the study design and protocol. Due to the nature of reminiscence therapy as a nonpharmacological intervention, it is not possible for the study participants or key study personnel to be blinded (Ito et al., 2007), so we evaluated all 24 RCTs as "high risk" of bias. In evaluation of the blinding of outcome assessment, we evaluated whether the measurement of outcome variables was performed by a third person and determined that 15 out of 24 RCTs were considered to have "low risk" of bias.

For evaluating incomplete outcome data, 11 out of 24 RCTs were evaluated as "low risk" of bias since more than $90 \%$ of the enrolled participants were included in the analysis. Studies with a low risk of bias were analyzed with the intention to treat (ITT) principal, mean value (Azcurra, 2012; Wang, 2007), or multiple imputation (O'Shea et al., 2014; Woods et al., 2012) in order to replace missing data.

The evaluation of reporting of selective outcomes was divided into either reported results or unreported results. Five out of 24 RCTs were evaluated as "high risk" of bias because the studies did not report results on all outcome variables mentioned in the methods section.

Overall risk of bias was evaluated, and studies with low risk of bias were considered as good quality studies if they included post-treatment assessment by assessors blind to treatment allocation (Woods et al., 2005). We also considered other bias such as detection, attrition, and reporting bias. Ultimately, 18 studies were evaluated to be of good quality.

\section{Statistical analysis}

A meta-analysis of 23 datasets from 24 studies was performed using Comprehensive Meta-Analysis Software $^{\circledR}$ (Biostat, Englewood, NJ, USA) to estimate the overall effect size of the intervention. Among the 24 studies included in the ROB assessment, one was excluded because it did not report statistical information such as the mean and standard deviation, which were required to calculate the effect size for the meta-analysis. We divided the result of one study according to the type of dementia and showed that the separated results did not violate the independence assumption (Tadaka and Kanagawa, 2007). The overall effect size was determined using standardized mean differences (SMDs) and $95 \%$ confidence intervals. A test for quantitative heterogeneity was performed using Cochran's $Q$ statistics based on Chi-square test. According to the result of Cochran's Q statistics, a random effect model was used, because Chi-square test $(p<0.1)$ indicated that the true effect size was not the same for all studies (Higgins et al., 2003). In addition, we computed $I^{2}$ as the percentage of overall variance from the heterogeneity among the studies. According to $I^{2}$ value, we confirmed the use of a random effect model, because an $I^{2}$ value larger than $50 \%$ indicated heterogeneity among included studies (Higgins et al., 2003). Funnel plots were generated to confirm the publication bias.

\section{Results}

\section{Description of the included studies}

Among 1763 participants in the 23 studies, the average ages of subjects in the intervention and control group were 85.2 and 85.3 years, respectively. Three studies included only Alzheimer's disease, two studies included only vascular disease, one study included both Alzheimer's and vascular dementia, and the rest of the studies included all types of dementia. Most of the studies screened the severity of dementia using either the MMSE or CDR and excluded subjects who had communication problems because of vision and/or hearing impairments.

In seven of the studies, the interventions were conducted in 12 sessions, which was the highest intervention dosage. Individual reminiscence interventions were conducted in five studies, while the rest of the studies conducted group reminiscence therapy. Interventions using social contact or counselling, exercise therapy, or usual care were included in the control group. Depression, cognition, behavioral problems, QoL, communication ability, functional status, and caregiver burden were assessed to measure the effects of the interventions. The results of the included studies are shown in Table 1.

\section{Effects of the interventions}

Depression, quality of life, and BPSD were selected to measure the effects of reminiscence therapy. Depression was measured in 16 RCTs. In 10 of these RCTs, depression was measured by the self-reported GDS, and the remaining 6 RCTs used the CSDD as an observed scale. With regard to the effects of reminiscence therapy on depression, Cochran's $Q$ was $91.37(p<0.001)$, and $I^{2}$ value was $84 \%$. The results indicated large heterogeneity, so a random effect model was used. The overall mean effect size for depression was -0.541 (95\% CI: -0.847 to $-0.234, \mathrm{Z}=-3.730, p<0.001)$, indicating that depression was significantly decreased in the reminiscence group compared to the control group.

Based on the moderate heterogeneity indicated by the Cochran's Q statistics $(p<0.1)$ and $I^{2}$ values $(>50 \%)$ for the variables of QoL, and BPSD, a random effect model was used. The overall mean effect size from 11 RCTs including QoL was 0.376 
Table 1. Summary of the included studies

\begin{tabular}{|c|c|c|c|c|c|}
\hline REFERENCE & $\begin{array}{l}\text { LEVEL OF } \\
\text { EVIDENCE }\end{array}$ & PARTICIPANTS & INTERVENTION & VARIABLES & RESULTS \\
\hline Akanuma et al. (2011) & + & $\begin{array}{l}\text { Japan } \\
n=24 \\
\text { Age: } 78.4(4.3) \\
\quad \text { for intervention group, } \\
78.1 \text { (5.3) for control group } \\
\text { Type of dementia: all VD }\end{array}$ & $\begin{array}{l}12 \text { weekly sessions } \\
\text { Intervention group }(n=12) \text { : } \\
\text { group RT with reality orientation } \\
\text { Control group }(n=12) \text { : } \\
\text { Only supportive care }\end{array}$ & $\begin{array}{l}\text { Cognition: MMSE } \\
\text { Affection: GDS } \\
\text { Behavior: BRSE } \\
\text { Social engagement: } \\
\quad \text { social activity }\end{array}$ & $\begin{array}{l}\text { Significant difference in } \\
\text { groups by time for } \\
\text { social activity } \\
(\mathrm{F}=38.568, \mathrm{p}<0.001)\end{array}$ \\
\hline Azcurra (2012) & + & $\begin{array}{l}\text { Argentina } \\
n=90 \\
\text { Age: } 85.3 \text { (5.6) for } \\
\text { intervention group, } 85.8 \\
\quad \text { (5.1) for control group } \\
\text { Type of dementia: AD }\end{array}$ & $\begin{array}{l}\text { Bi-weekly sessions } \\
\text { for } 12 \text { weeks } \\
\text { Intervention group }(n=45) \text { : } \\
\text { group RT } \\
\text { Control group }(n=45) \text { : } \\
\text { counseling and } \\
\text { informal social contact }\end{array}$ & $\begin{array}{l}\text { Functional status: ADL } \\
\text { QoL: SRQoL, WIB } \\
\text { Social engagement: SES } \\
\text { Carer: ZBI }\end{array}$ & $\begin{array}{l}\text { Significant differences in } \\
\text { groups by time for } \\
\text { QoL }(\mathrm{F}=14.7, \mathrm{p}<0.01) \\
\text { and SES }(\mathrm{F}=7.5, \mathrm{p}<0.01) \text {. }\end{array}$ \\
\hline Baines et al. (1987) & + & $\begin{array}{l}\text { UK } \\
n=10 \\
\text { Age: } 82.7 \text { for intervention group, } \\
\quad 80.4 \text { for control group } \\
\text { Type of dementia: all types }\end{array}$ & $\begin{array}{l}4 \text { weekly sessions of } 30 \mathrm{~min} \\
\text { Intervention group }(n=5) \text { : } \\
\text { group RT } \\
\text { Control group }(n=5) \text { : } \\
\text { no treatment }\end{array}$ & $\begin{array}{l}\text { Cognition: CAS } \\
\text { Behavior: CAPE behavior } \\
\text { rating scale, problem } \\
\text { behavior rating scale } \\
\text { QoL: life satisfaction indices } \\
\text { Social engagement: Holden } \\
\text { communication scale } \\
\text { Carer: staff knowledge }\end{array}$ & $\begin{array}{l}\text { Reminiscence group slightly } \\
\text { improved in life satisfaction, } \\
\text { but not significant. }\end{array}$ \\
\hline Chang and Je (2008) & - & $\begin{array}{l}\text { Korea } \\
n=30 \\
\text { Age: } 77.8 \text { for intervention group, } \\
76.0 \text { for control group } \\
\text { Type of dementia: all types }\end{array}$ & $\begin{array}{l}8 \text { weekly sessions } \\
\text { Intervention group }(n=15) \text { : } \\
\text { group RT } \\
\text { Control group }(n=15) \text { : } \\
\text { supportive care }\end{array}$ & $\begin{array}{l}\text { Cognition: MMSE } \\
\text { Affection: GDS } \\
\text { QoL: GQoL-D }\end{array}$ & $\begin{array}{l}\text { Significant differences in } \\
\text { groups by time for MMSE } \\
\begin{array}{l}(\mathrm{F}=5.10, \mathrm{p}<0.05), \mathrm{GDS} \\
(\mathrm{F}=9.15, \mathrm{p}<0.001) \text {, and } \\
\text { QoL }(\mathrm{F}=16.31, \mathrm{p}<0.001\end{array}\end{array}$ \\
\hline Chang and Lee (2006) & - & $\begin{array}{l}\text { Korea } \\
n=24 \\
\text { Age: } 78.8 \text { for intervention group, } \\
\quad 79.3 \text { for control group } \\
\text { Type of dementia: all types }\end{array}$ & $\begin{array}{l}8 \text { weekly sessions } \\
\text { Intervention group }(n=10) \text { : } \\
\text { group RT } \\
\text { Control group }(n=14) \text { : } \\
\text { supportive care }\end{array}$ & $\begin{array}{l}\text { Affection: GDS } \\
\text { QoL: GQoL-D } \\
\text { Social engagement: SBS } \\
\text { social behavior scale }\end{array}$ & $\begin{array}{l}\text { GDS }(t=4.28, p<0.01) \\
\text { and social engagement } \\
(t=-3.68, p<0.01), \text { QoL } \\
(t=2.51, p<0.05) \text { were } \\
\text { significantly different } \\
\text { between pre- and post-test } \\
\text { in the reminiscence group. }\end{array}$ \\
\hline
\end{tabular}




\section{Table 1. Continued}

\begin{tabular}{|c|c|c|c|c|c|}
\hline REFERENCE & $\begin{array}{l}\text { LEVEL OF } \\
\text { EVIDENCE }\end{array}$ & PARTICIPANTS & INTERVENTION & VARIABLES & RESULTS \\
\hline $\begin{array}{l}\text { Duru Aşiret and } \\
\text { Kapucu (2016) }\end{array}$ & + & $\begin{array}{l}\text { Turkey } \\
n=62 \\
\text { Age: } 81.8(4.8) \\
\quad \text { for intervention group, } \\
82.2(5.0) \text { for control group } \\
\text { Type of dementia: AD }\end{array}$ & $\begin{array}{l}12 \text { weekly sessions of } 30-40 \mathrm{~min} \\
\text { Intervention group }(n=31) \text { : } \\
\text { individual RT } \\
\text { Control group }(n=31) \text { : usual care }\end{array}$ & $\begin{array}{l}\text { Cognition: MMSE } \\
\text { Affection: GDS } \\
\text { Functional status: ADL }\end{array}$ & $\begin{array}{l}\text { Significant differences in } \\
\text { groups by time for MMSE } \\
(\mathrm{F}=22.418, \mathrm{p}<0.001) \\
\text { and GDS }(\mathrm{F}=30.518 \\
\mathrm{p}<0.001)\end{array}$ \\
\hline Goldwasser et al. (1987) & - & $\begin{array}{l}\text { USA } \\
n=18 \\
\text { Age: } 81.6 \text { for intervention group, } \\
\quad 83.1 \text { for control group } \\
\text { Type of dementia: all types }\end{array}$ & $\begin{array}{l}10 \text { sessions over } 5 \text { weeks } \\
\text { Intervention group }(n=9) \text { : } \\
\text { group RT } \\
\text { Control group }(n=9): \text { no } \\
\text { treatment }\end{array}$ & $\begin{array}{l}\text { Affection: Beck } \\
\text { depression scale } \\
\text { Functional status: } \\
\text { Katz index (ADL) }\end{array}$ & $\begin{array}{l}\text { Significant differences in } \\
\text { groups by time for } \\
\text { depression }(\mathrm{F}=3.38 \\
\mathrm{p}=<0.05)\end{array}$ \\
\hline Haight et al. (2006) & - & $\begin{array}{l}\text { USA } \\
n=30 \\
\text { Age: range } 60-99 \\
\text { Type of dementia: all types }\end{array}$ & $\begin{array}{l}6 \text { weekly sessions } \\
\text { Intervention group }(n=15) \text { : } \\
\text { group RT } \\
\text { Control group }(n=15) \text { : usual care }\end{array}$ & $\begin{array}{l}\text { Cognition: MMSE } \\
\text { Affection: depression } \\
\quad \text { (CSDD), AMS } \\
\text { Behavior: MBS } \\
\text { Functional status: FIM } \\
\text { Social engagement: COS }\end{array}$ & $\begin{array}{l}\text { Significant differences in } \\
\text { groups by time for MMSE } \\
\begin{array}{l}(\mathrm{F}=20.77, \mathrm{p}=0.0005) \\
\text { CSDD }(\mathrm{F}=7.54, \\
\mathrm{p}=0.015), \text { Alzheimer's } \\
\text { Mood Scale }(\mathrm{F}=9.47, \\
\mathrm{p}=0.008) \text { (only good } \\
\text { mood), and } \\
\text { Communication } \\
\text { Observation Scale } \\
(\mathrm{F}=23.36, \mathrm{p}=0.005) .\end{array}\end{array}$ \\
\hline Hilgeman et al. (2014) & + & $\begin{array}{l}\text { USA } \\
n=18 \\
\text { Age: } 80.8(4.4) \text { for intervention } \\
\text { group, } 84.2(7.9) \text { for control group } \\
\text { Type of dementia: all types }\end{array}$ & $\begin{array}{l}4 \text { weekly sessions } \\
\text { Intervention group }(n=10) \text { : } \\
\text { individual reminiscence } \\
\text { Control group }(n=8) \text { : minimal } \\
\text { intervention via telephone }\end{array}$ & $\begin{array}{l}\text { Cognition: DCS, IMMEL } \\
\text { Affection: Depression } \\
\quad \text { (CSDD) } \\
\text { QoL: QoL-AD, EQ-5D } \\
\text { Social engagement: Social } \\
\quad \text { engagement } \\
\text { Carer: CSDD, EQ-5D }\end{array}$ & $\begin{array}{l}\text { Depression }(\mathrm{F}=5.50, \\
\mathrm{p}=0.03) \text { was significantly } \\
\text { different in the reminiscence } \\
\text { group. }\end{array}$ \\
\hline Hsieh et al. (2010) & - & $\begin{array}{l}\text { Taiwan } \\
n=61 \\
\text { Age: } 77.5(8.4) \text { for intervention } \\
\quad \text { group, } 77.2(10.4) \text { for } \\
\text { control group } \\
\text { Type of dementia: all types }\end{array}$ & $\begin{array}{l}12 \text { weekly sessions of } 40-50 \mathrm{~min} \\
\text { Intervention group }(n=29) \text { : } \\
\text { group RT } \\
\text { Control group }(n=32) \text { : usual care }\end{array}$ & $\begin{array}{l}\text { Cognition: AES } \\
\text { Affection: GDS } \\
\text { Behavior: NPI }\end{array}$ & $\begin{array}{l}\text { Significant differences in } \\
\text { groups by time for GDS } \\
(Z=-2.99, p=0.003) \text { and } \\
\text { NPI }(Z=-2.20, p=0.028) .\end{array}$ \\
\hline Ito et al. (2007) & + & $\begin{array}{l}\text { Japan } \\
n=35 \\
\text { Age: } 82.9(6.4) \text { for intervention } \\
\text { group, } 82.1(5.2) \text { for } \\
\quad \text { control group } \\
\text { Type of dementia: VD }\end{array}$ & $\begin{array}{l}12 \text { weekly sessions } \\
\text { Intervention group }(n=17) \text { : } \\
\text { group RT and reality orientation } \\
\text { Control group }(n=18) \text { : usual care }\end{array}$ & $\begin{array}{l}\text { Cognition: MMSE, CASI } \\
\text { Functional status: MOSES }\end{array}$ & No significant improvement. \\
\hline
\end{tabular}




\begin{tabular}{|c|c|c|c|c|c|}
\hline REFERENCE & $\begin{array}{l}\text { LEVEL OF } \\
\text { EVIDENCE }\end{array}$ & PARTICIPANTS & INTERVENTION & VARIABLES & RESULTS \\
\hline Kwon et al. (2012) & + & $\begin{array}{l}\text { Korea } \\
n=20 \\
\text { Age: } 81.4(4.1) \text { for intervention } \\
\text { group, } 79.9(9.3) \text { for } \\
\text { control group } \\
\text { Type of dementia: all types }\end{array}$ & $\begin{array}{l}20 \text { sessions over } 7 \text { weeks } \\
\text { Intervention group }(n=10) \text { : } \\
\text { group RT } \\
\text { Control group }(n=10) \text { : } \\
\text { exercise program }\end{array}$ & Social engagement: SBS & $\begin{array}{l}\text { Social communication by } \\
\text { verbal subscale }(Z=-2.83 \text {, } \\
p=0.005) \text { and by non- } \\
\text { verbal subscale }(Z=-2.44, \\
p=0.015) \text { were significantly } \\
\text { different in the reminiscence } \\
\text { group. }\end{array}$ \\
\hline Lai et al. (2004) & + & $\begin{array}{l}\text { Hong Kong } \\
n=66 \\
\text { Age: } 86.2(6.3) \text { for intervention } \\
\text { group, } 86.8(7.3) \text { for } \\
\text { control group } \\
\text { Type of dementia: all types }\end{array}$ & $\begin{array}{l}6 \text { weekly sessions for } 30 \mathrm{~min} \\
\text { Intervention group }(n=36) \text { : } \\
\text { group RT } \\
\text { Control group }(n=30) \text { : usual care }\end{array}$ & $\begin{array}{l}\text { Functional status: functional } \\
\text { abilities, MDS-ADL } \\
\text { QoL: Well-being/Ill-being } \\
\text { Scale } \\
\text { Social engagement: SES }\end{array}$ & $\begin{array}{l}\text { Social engagement }(\mathrm{p}=0.032) \\
\text { and well-being }(\mathrm{p}=0.014) \\
\text { were significantly different } \\
\text { between pre and post in the } \\
\text { reminiscence group. }\end{array}$ \\
\hline $\begin{array}{l}\text { Morgan and Woods } \\
\quad(2012)\end{array}$ & + & $\begin{array}{l}\text { UK } \\
n=17 \\
\text { Age: } 80.5(5.7) \text { for intervention } \\
\quad \text { group, } 84.4(7.8) \text { for } \\
\quad \text { control group } \\
\text { Type of dementia: all types }\end{array}$ & $\begin{array}{l}12 \text { or more weekly sessions } \\
\text { of } 30-60 \text { min } \\
\text { Intervention group }(n=8) \text { : } \\
\text { individual reminiscence } \\
\text { Control group }(n=9) \text { : usual care }\end{array}$ & $\begin{array}{l}\text { Cognition: memory (AMI } \\
\text { with AIS and PSS } \\
\text { subscale) } \\
\text { Affection: depression } \\
\text { (GDS-SF) }\end{array}$ & $\begin{array}{l}\text { No statistical difference was } \\
\text { found. }\end{array}$ \\
\hline Nakamae et al. (2014) & - & $\begin{array}{l}\text { Japan } \\
n=30 \\
\text { Age: } 84.7(6.8) \text { for intervention } \\
\text { group, } 87.1(4.5) \text { for } \\
\text { control group } \\
\text { Type of dementia: all types }\end{array}$ & $\begin{array}{l}6 \text { weekly sessions of } 40 \mathrm{~min} \\
\text { Intervention group }(n=15) \text { : } \\
\text { group RT (making and eating } \\
\text { a rice ball while reminiscing) } \\
\text { Control group }(n=15) \text { : only } \\
\text { eating a rice ball }\end{array}$ & $\begin{array}{l}\text { Cognition: MMSE } \\
\text { Affection: CSDD } \\
\text { Behavior: MOSES } \\
\quad \text { (irritability, withdrawal) } \\
\text { Functional status: MOSES } \\
\text { (self-care) } \\
\text { Social engagement: } \\
\text { communication } \\
\text { (Vitality Index) }\end{array}$ & $\begin{array}{l}\text { CSDD }(\mathrm{p}<0.05) \text { was } \\
\text { significantly different } \\
\text { between the pre- and } \\
\text { post-tests only in the } \\
\text { reminiscence group. }\end{array}$ \\
\hline Nakatsuka et al. (2015) & + & $\begin{array}{l}\text { Japan } \\
n=82 \\
\text { Age: } 81.2(4.2) \\
\text { for intervention group, } \\
81.3 \text { (3.8) for control group } \\
\text { Type of dementia: mild cognitive } \\
\text { impairment or } 0.5 \text { points } \\
\text { on the CDR }\end{array}$ & $\begin{array}{l}12 \text { weekly sessions }+12 \text { home } \\
\text { assignments for } 60 \text { min } \\
\text { Intervention group }(n=44) \text { : } \\
\text { group RT } \\
\text { Control group }(n=38) \text { : } \\
\text { physical activities }\end{array}$ & $\begin{array}{l}\text { Cognition: MMSE, } \\
\text {-TMT-A Affection: GDS } \\
\text { Functional status: physical } \\
\text { function, } \\
\text { 6-meter walk time } \\
\text { Social engagement: word } \\
\text { fluency (WF) } \\
\text { QoL: QoL Face Scale scores }\end{array}$ & $\begin{array}{l}\text { MMSE, WF, and QoL } \\
\text { increased in both groups, } \\
\text { and GDS decreased in both } \\
\text { groups. No significant } \\
\text { differences were found. }\end{array}$ \\
\hline
\end{tabular}


Table 1. Continued

\begin{tabular}{|c|c|c|c|c|c|}
\hline REFERENCE & $\begin{array}{l}\text { LEVEL OF } \\
\text { EVIDENCE }\end{array}$ & PARTICIPANTS & INTERVENTION & VARIABLES & RESULTS \\
\hline O'Shea et al. (2014) & + & $\begin{array}{l}\text { Ireland } \\
n=304 \\
\text { Age: } 85.2(7.1) \text { for intervention } \\
\text { group, } 85.7(7.1) \text { for control group } \\
\text { Type of dementia: all types }\end{array}$ & $\begin{array}{l}18 \text { weekly sessions } \\
\text { Intervention group }(n=153) \text { : } \\
\text { group RT } \\
\text { Control group }(n=151) \text { : } \\
\text { usual care }\end{array}$ & $\begin{array}{l}\text { Affection: CSDD } \\
\text { Behavior: agitation (CMAI) } \\
\text { QoL: self-reported } \\
\text { QoL-AD } \\
\text { Carer: MZBI }\end{array}$ & $\begin{array}{l}\text { Significant differences in } \\
\text { groups by time for CSDD } \\
(p=0.03) \text { and QoL-AD } \\
(p=0.04) \text {. }\end{array}$ \\
\hline $\begin{array}{l}\text { Subramaniam } \\
\text { et al. }(2014)\end{array}$ & + & $\begin{array}{l}\text { UK } \\
n=23 \\
\text { Age: } 84.5(6.7) \text { for intervention } \\
\quad \text { group, } 88.3(6.0) \text { for } \\
\text { control group } \\
\text { Type of dementia: all types }\end{array}$ & $\begin{array}{l}\text { 11-16 weekly sessions } \\
\text { Intervention group }(n=11) \text { : } \\
\text { individual reminiscence } \\
\text { Control group }(n=12) \text { : } \\
\text { usual care }\end{array}$ & $\begin{array}{l}\text { Cognition: AMI } \\
\text { Affection: GDS-12R } \\
\text { QoL: QoL-AD } \\
\text { Social engagement: social } \\
\text { relationships, QCPR } \\
\text { Carer: staff attitudes, ADQ, } \\
\text { staff knowledge }\end{array}$ & $\begin{array}{l}\text { Significant differences in } \\
\text { groups by time for } Q o L \\
(F=5.11, p=0.035) \text { and } \\
\text { cognition }(F=19.92, \\
p<0.001)\end{array}$ \\
\hline $\begin{array}{l}\text { Tadaka and Kanagawa } \\
\text { (2007) }\end{array}$ & + & $\begin{array}{l}\text { Japan } \\
n=60 \\
\text { Age: } 81.2(6.2) \text { for intervention } \\
\text { group, } 85.3(6.3) \text { for } \\
\text { control group } \\
\text { Type of dementia: all types }\end{array}$ & $\begin{array}{l}8 \text { weekly sessions } \\
\text { Intervention group }(n=30) \text { : } \\
\text { group RT } \\
\text { Control group }(n=30) \text { : } \\
\text { usual care }\end{array}$ & $\begin{array}{l}\text { Cognition: MMSE } \\
\text { Affection: depression } \\
\text { Behavior: disorientation, } \\
\text { irritability with withdrawal } \\
\text { Functional status: ADL }\end{array}$ & $\begin{array}{l}\text { Withdrawal in AD } \\
(p<0.05) \text { and } \\
\text { cognitive function in VD } \\
(\mathrm{p}<0.05) \text { were significantly } \\
\text { different. }\end{array}$ \\
\hline $\begin{array}{l}\text { Thorgrimsen } \\
\text { et al. (2002) }\end{array}$ & + & $\begin{array}{l}\text { UK } \\
n=11 \\
\text { Age: } 76.9(4.6) \text { for intervention } \\
\text { group, } 75.5(7.1) \text { for } \\
\text { control group } \\
\text { Type of dementia: all types }\end{array}$ & $\begin{array}{l}18 \text { weekly sessions } \\
\text { Intervention group }(n=7) \text { : } \\
\text { group RT } \\
\text { Control group }(n=4) \text { : usual care }\end{array}$ & $\begin{array}{l}\text { Cognition: MMSE } \\
\text { Behavior: CAPE-BRS } \\
\text { QOL: QOL-AD } \\
\text { Social engagement: Holden } \\
\quad \text { communication scale } \\
\text { Carer: GHQ-12, stress (RSS) }\end{array}$ & $\begin{array}{l}\text { No statistical difference } \\
\text { was found. }\end{array}$ \\
\hline van Bogaert et al. (2013) & + & $\begin{array}{l}\text { Belgium } \\
n=82 \\
\text { Age: } 83 \text { for intervention } \\
\text { group, } 85 \text { for control group } \\
\text { Type of dementia: AD or probable } \\
\text { AD }\end{array}$ & $\begin{array}{l}8 \text { sessions over } 4 \text { weeks } \\
45 \text { min for each session } \\
\text { Intervention group }(n=41) \text { : } \\
\text { individual reminiscence } \\
\text { Control group }(n=41) \text { : } \\
\text { usual care }\end{array}$ & $\begin{array}{l}\text { Cognition: MMSE, FAB } \\
\text { Affection: GDS-30, CSDD } \\
\text { Behavior: NPI }\end{array}$ & $\begin{array}{l}\text { MMSE }(\mathrm{p}<0.05), \text { GDS } \\
(\mathrm{p}<0.001) \text {, and CSDD } \\
(\mathrm{p}<0.05) \text { were significantly } \\
\text { different between pre and } \\
\text { post-test in the reminiscence } \\
\text { group. }\end{array}$ \\
\hline Wang (2007) & + & $\begin{array}{l}\text { Taiwan } \\
n=102 \\
\text { Age: } 79.7(6.2) \text { for intervention } \\
\text { group, } 78.9(7.6) \text { for } \\
\text { control group } \\
\text { Type of dementia: all types }\end{array}$ & $\begin{array}{l}8 \text { weekly sessions of } 1 \text { hour } \\
\text { Intervention group }(n=51) \text { : } \\
\text { group reminiscence } \\
\text { Control group }(n=51) \text { : } \\
\text { usual care }\end{array}$ & $\begin{array}{l}\text { Cognition: MMSE } \\
\text { Affection: GDS-SF, CSDD }\end{array}$ & $\begin{array}{l}\text { Significant differences in } \\
\text { groups by time for MMSE } \\
(\mathrm{F}=6.16, \mathrm{p}=0.015) \text { and } \\
\text { CSDD }(\mathrm{F}=5.13, \mathrm{p}=0.026) \text {. }\end{array}$ \\
\hline
\end{tabular}




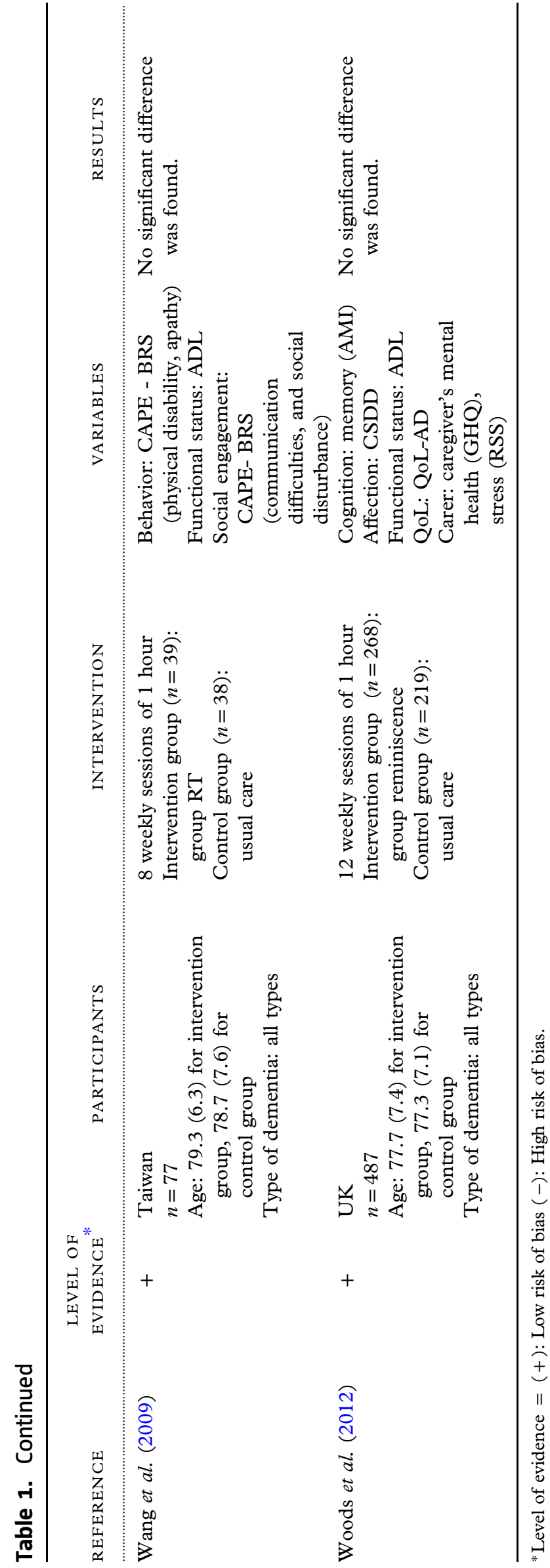

(95\% CI: 0.075 to $0.677, \mathrm{Z}=2.452, p=0.014$ ), and the overall mean effect size from 10 RCTs including the BPSD variable was $-0.327(95 \%$ CI: -0.591 to $-0.64, \mathrm{Z}=-2.435, p=0.015$ ). These results indicate that QoL significantly increased and BPSD significantly decreased in the reminiscence group compared to the control group. The results comparing depression, QoL, and BPSD between the reminiscence and control group are presented in Figures 3-5.

Sub-group analysis was conducted to compare the results from studies with high quality or studies with small sample size and the results of pooled analysis from 24 RCTs. The result for 18 studies with high quality presented a small to medium effect size $(p<0.05)$, which was similar to the result of pooled analysis from 24 RCTs except for QoL $(d=0.23,95 \%$ CI: -0.054 to $0.53, \mathrm{Q}=30.87$, $\left.p<0.001, \mathrm{I}^{2}=74.08\right)$. In the result from 11 studies with small sample size $(\leq 50)$, the effect size for cognition and BPSD showed homogeneity, and the effect size for depression, QoL, and BPSD was small $(p<0.05)$. On the contrary, in 13 studies with large sample size $(>50)$, the effect sizes for depression was significantly different, while those for BPSD and QoL were not significantly different between groups.

With regard to the subgroup analysis for group versus individual reminiscence therapy, the overall mean effect size of the depression variable was -0.785 (95\% CI: -1.191 to $-0.378, Z=-5.451$, $p<0.001$ ) from 5 RCTs that conducted individual reminiscence therapy, and -0.466 (95\% CI: -0.798 to $-0.135, \mathrm{Z}=-2.759, p=0.006)$ from $12 \mathrm{RCTs}$ that conducted group reminiscence therapy.

A funnel plot method was conducted to assess publication bias. The distribution of studies examining depression, and QoL had a symmetric shape in the funnel plot, indicating that these results did not have any publication bias. However, the distribution of studies on BPSD showed an asymmetric shape, so Kendall's tau statistic was calculated (Begg and Mazumdar, 1994). Kendall's tau with continuity correction was $-0.53(\mathrm{z}=2.14, p=0.03)$. Additionally, the imputed point estimate was $0.013(95 \%$ CI: -0.258 to 0.285 ) using the trim and fill method, presenting that 6 studies are missing. Therefore, these statistics indicated publication bias in the studies included to calculate the effect size for BPSD. Funnel plots are presented in Figure 6.

\section{Discussion}

\section{Assessment of the risk of bias}

This study conducted a systematic review and metaanalysis to examine the effect of reminiscence therapy; however, there were some issues with the 


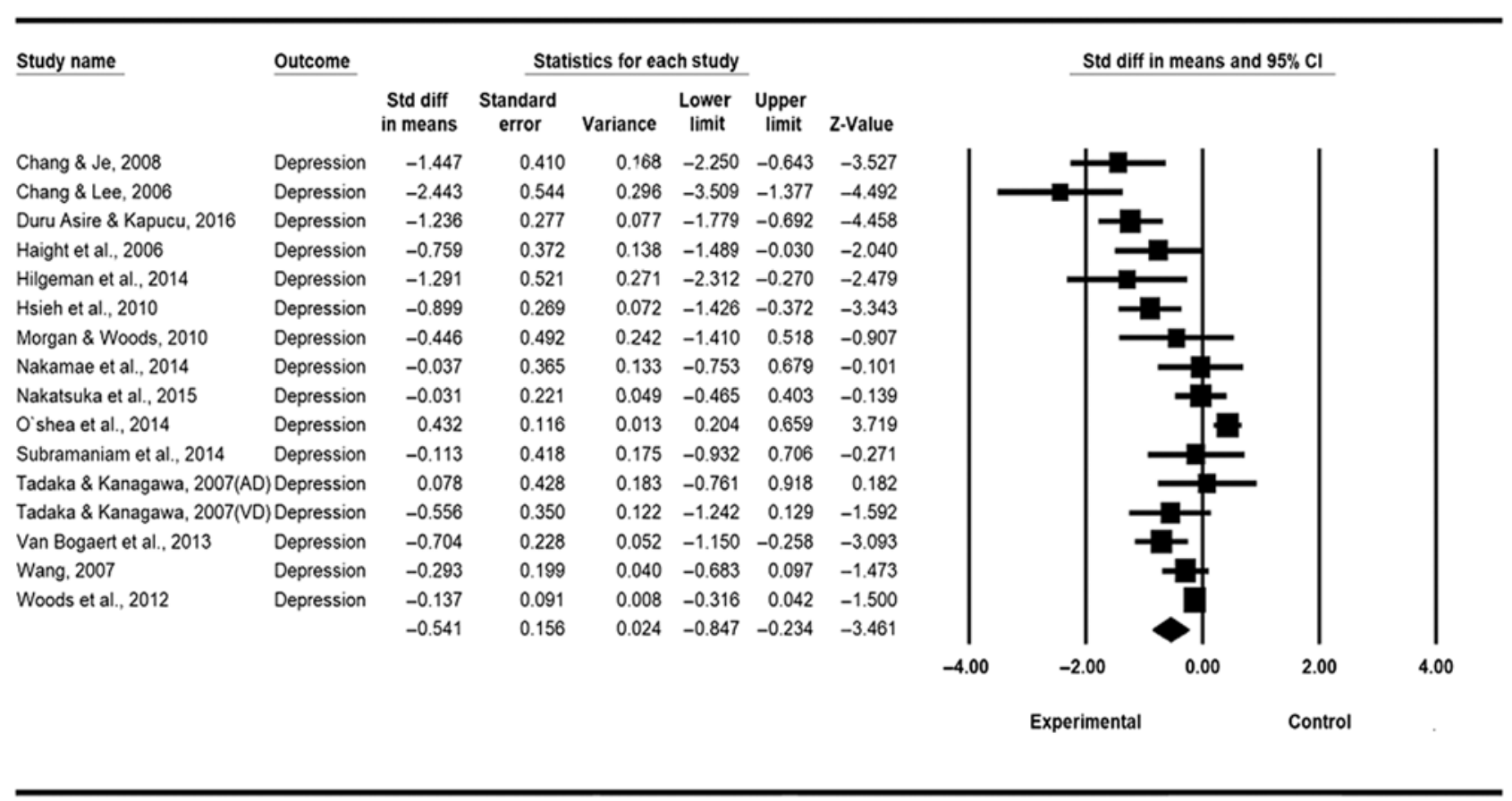

Figure 3. Comparison outcomes of reminiscence therapy versus control: depression.

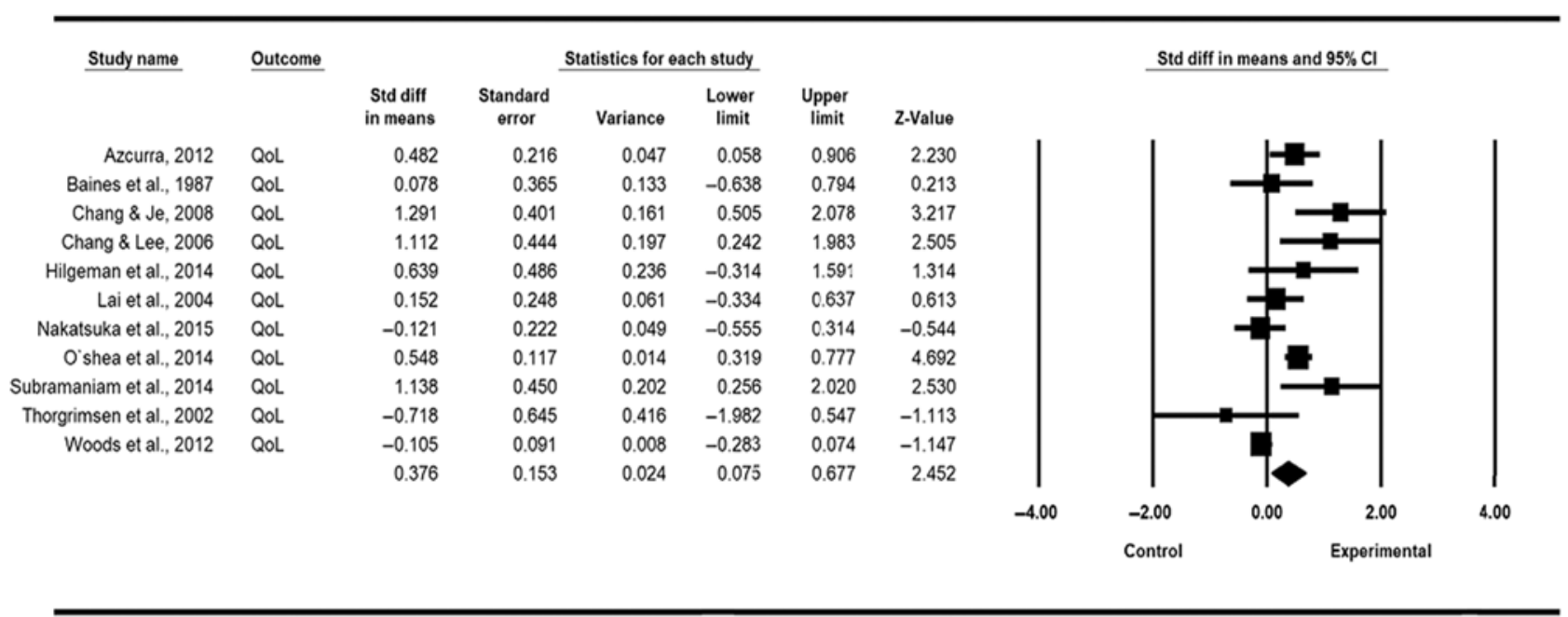

Figure 4. Comparison outcomes of reminiscence therapy versus control: quality of life.

assessment of the risk of bias. The first issue was regarding the interpretation between the evaluation of "Risk of Bias" as qualitative information and the overall effect size as quantitative information calculated by the meta-analysis. In terms of the qualitative evaluation, only 18 out of the 24 RCTs had a low risk of bias (high methodological quality), and the rest had a high risk of bias. Thirteen of the studies had a large sample size more than 50 participants. There were only 2 trials which have more than 300 participants. Two trials that had a very large sample size $(>300)$ or low risk of bias failed to identify significant differences between the reminiscence group and control group (O'Shea et al., 2014; Woods et al., 2012).

The result of meta-analysis is retrospective and is susceptible to several sources of bias. Studies with low methodological quality might have been included in the meta-analyses and could have altered the interpretation of the benefit of intervention. Therefore, it is very important to assess the quality of individual studies before performing metaanalysis (Chalmers, 1991; Felson, 1992). This current analysis only included studies belong to the inclusion criteria after conducting assessment of trial quality with ROB tool developed by the Cochrane 


\begin{tabular}{|c|c|c|c|c|c|c|c|c|c|c|c|}
\hline \multirow[t]{2}{*}{$\underline{\text { Study name }}$} & \multirow[t]{2}{*}{ Outcome } & \multicolumn{6}{|c|}{ Statistics for each study } & & \multicolumn{2}{|c|}{ Std diff in means and $95 \% \mathrm{Cl}$} & \\
\hline & & $\begin{array}{l}\text { Std diff } \\
\text { in means }\end{array}$ & $\begin{array}{l}\text { Standard } \\
\text { error }\end{array}$ & Variance & $\begin{array}{l}\text { Lower } \\
\text { limit }\end{array}$ & $\begin{array}{l}\text { Upper } \\
\text { limit }\end{array}$ & \multicolumn{2}{|l|}{ Z-Value } & & & \\
\hline Haight et al., 2006 & BPSD & -0.658 & 0.375 & 0.141 & -1.393 & 0.077 & -1.756 & & & & \\
\hline Hsieh et al., 2010 & BPSD & -0.519 & 0.261 & 0.068 & -1.029 & -0.008 & -1.990 & & & & \\
\hline Nakamae et al., 2014 & BPSD & -0.169 & 0.366 & 0.134 & -0.886 & 0.547 & -0.463 & & & & \\
\hline Tadaka \& Kanagawa, 2007(AD) & BPSD & -1.071 & 0.458 & 0.209 & -1.968 & -0.174 & -2.341 & & & & \\
\hline Tadaka \& Kanagawa, 2007(VD) & BPSD & -0.560 & 0.350 & 0.122 & -1.245 & 0.125 & -1.602 & & & & \\
\hline Thorgrimsen et al., 2002 & BPSD & -1.346 & 0.689 & 0.475 & -2.697 & 0.005 & -1.952 & & & & \\
\hline Van Bogaert et al., 2013 & BPSD & -0.098 & 0.221 & 0.049 & -0.531 & 0.335 & -0.443 & & & & \\
\hline \multirow[t]{3}{*}{ Wang et al., 2009} & BPSD & -0.289 & 0.305 & 0.093 & -0.887 & 0.309 & -0.947 & & & & \\
\hline & & -0.327 & 0.134 & 0.018 & -0.591 & -0.064 & -2.435 & & & & \\
\hline & & & & & & & & -4.00 & -2.00 & 2.00 & 4.00 \\
\hline
\end{tabular}

Figure 5. Comparison outcomes of reminiscence therapy versus control: BPSD.
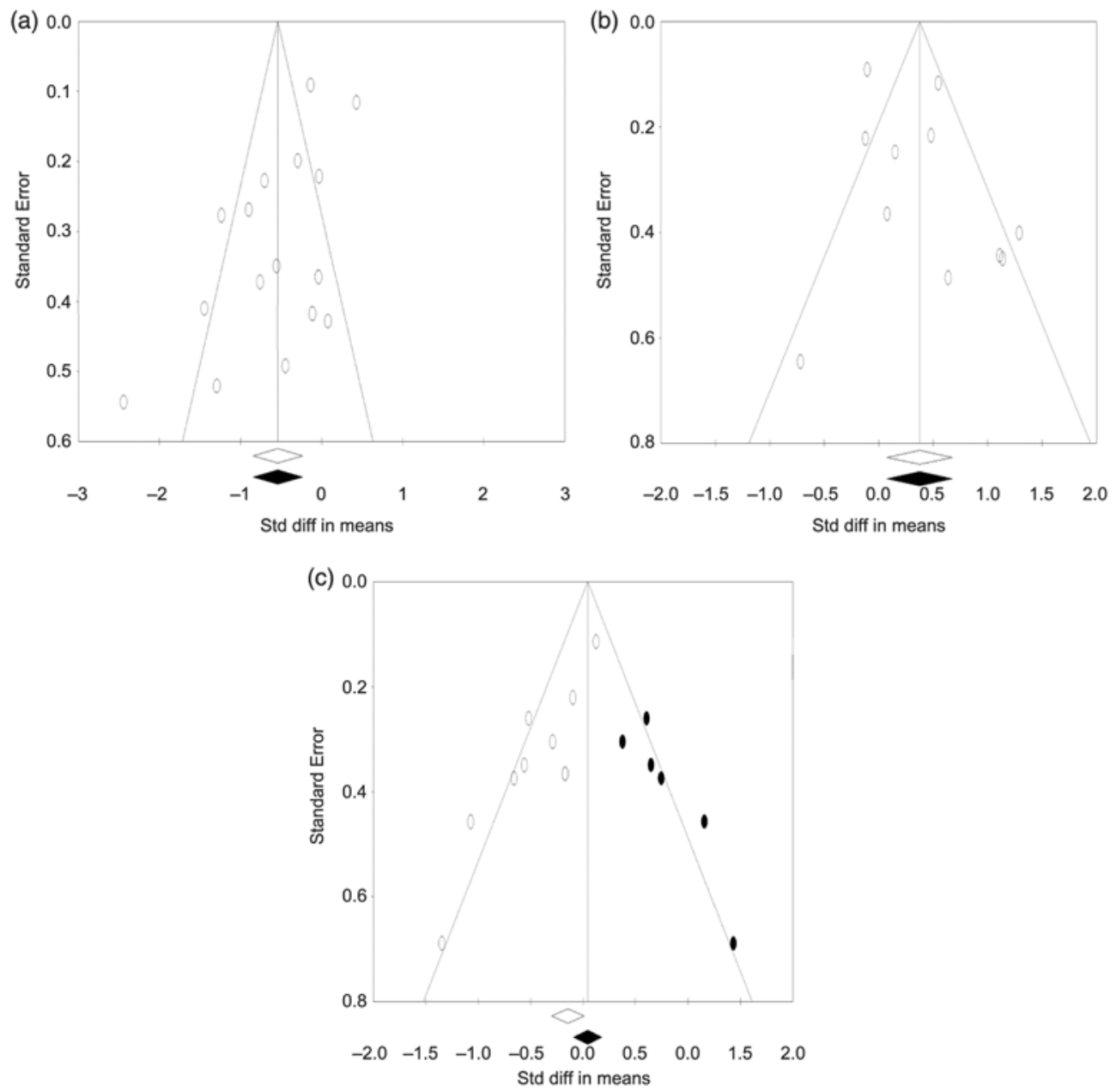

Figure 6. Funnel plot of selected studies for effect size extraction. $A=$ Depression; $B=$ Quality of Life; $C=B P S D$.

Collaboration. This current study only included RCTs in order to exclude low quality studies such as case control study, cross sectional study, and protocol study. Although we tried to exclude studies with low quality, we also had to consider publication bias; therefore, we included three articles published in Korean, even though they did not clearly describe random sequence generation and allocation concealment related to selection bias.

The effect size for depression, QoL, and BPSD from 11 studies with small sample size was small. On the contrary, in 13 studies with large sample size, the 
effect sizes for depression was significantly different, while those for BPSD and QoL were not significantly different between groups. Our result was different from those of previous RCTs with a very large sample size or well-designed structure (O'Shea et al., 2014; Woods et al., 2012), so there is some argument. The purpose of RCTs is to present a strong treatment effect through the least biased estimates of treatment. On the contrary, systematic review based on meta-analysis aims to integrate individual effect size, not to summarize (Bailar, 1997).

Secondly, in terms of attrition bias, some studies applied the ITT principle, while other studies analyzed per-protocol or did not have any missing cases. ITT analysis includes every subject that is randomized for treatment assignment (Gupta, 2011). The purpose of the ITT approach maintains that the treatment group is similar to the original randomly assigned groups (Hollis and Campbell, 1999). ITT analysis does not allow noncompliance, protocol deviations, or withdrawal and does not consider anything that occurs after randomization (Wertz, 1995). Thus, the Cochrane Handbook posits that, if ITT analysis was applied during a trial, attrition bias can be considered low (Hartling et al., 2012; Higgins and Altman, 2008). However, in ITT analysis, the estimate of treatment effect is generally conservative, and marked difference between compliance and non-compliance groups can complicate data interpretation. Therefore, ITT analysis has been criticized for being too cautious and more susceptible to type 2 error (Gupta, 2011; Hollis and Campbell, 1999). Woods et al. (2012) reported that their study examined the differences in the results between analysis using the ITT method and complete case analysis and found no significant differences. Furthermore, Woods et al. (2012) reported that compliance analysis was significantly different in memory, quality of relationships, and QoL between reminiscence and control groups. Other studies also reported significant differences in outcomes between the ITT method and per-protocol analysis (O'Shea et al., 2014; Tadaka and Kanagawa, 2007). Therefore, better application of ITT analysis is possible if complete outcome data are available for all randomized subjects (Gupta, 2011). Multiple imputation and maximum likelihood methods account for the missing data and the uncertainties they cause, and then a per-protocol technique could be used as a supportive method (CPMP, 2000). Because all of the trials used substandard methods with the potential for bias, we suggest a suitable method for dealing with missing data to increase the effects of non-pharmacological intervention, to reduce the attrition bias, and to increase the quality of evidence in RCTs.

\section{Effectiveness of reminiscence therapy in elderly patients with dementia}

Depression is the mostly commonly selected variable to identify the effectiveness of reminiscence therapy in individual studies on PWD. According to findings from this meta-analysis, reminiscence therapy for PWD had a medium effect size on depression, as demonstrated by significantly decreased depression in the reminiscence group compared to the control group. Depressed mood commonly occurs in PWD (Gottfries, 2001). Chin (2007) reported that reminiscence therapy also has effects on happiness and depression in older adults without dementia. The American Psychiatric Association (APA) has described four different psychotherapeutic approaches for guiding a reminiscence therapy practice to treat patients with dementia. One of these psychotherapeutic approaches is an emotion-oriented approach that aims to improve mood using reminiscence therapy (APA, 2007). For patients with mood disturbance, a systematic review of dementia practice guidelines recommended that nonpharmacological management such as reminiscence therapy should be applied first (Ngo and HolroydLeduc, 2014). The findings from the systematic review in this study are along the same lines as the APA description and dementia practice guidelines. This could be explained by the finding that the reduction in depressive symptoms could be related to other factors such as increased communication during the intervention. PWD were able to talk without being criticized while reminiscing, which increased their confidence after reminiscence therapy (Duru Aşiret and Kapucu, 2016). Therefore, the effectiveness of a non-pharmacological intervention should also consider that PWD are receiving a pharmacological intervention; furthermore, the non-pharmacological intervention should have a large effect on reducing the occurrence of depressive mood in PWD (van Bogaert et al., 2013).

Lai et al. (2004) reported that measuring changes in dementia patients is very difficult because there are limitations to addressing cognitive function or behavior on their own, so pleasure and wellbeing could be more suitable measures to identify the effects of interventions in the elderly with dementia. Nakatsuka et al. (2015) also reported that quality of life might be related to a good attitude and impressive experience during the intervention, so enjoyableness was important for the wellbeing of dementia patients regardless of the type of intervention. Stinson (2009) also reported that reminiscence therapy increased adaptation to the present time, quality of life, and satisfaction because this intervention is based on remembering events experienced in the past. Therefore, our result was consistent with the Stinson (2009) report. 
Behavioral problems are recognized as important clinical features of dementia. These problems decrease patient quality of life and increase caregiver burden (Akanuma et al., 2011). A systematic review for dementia practice guidelines recommended that pharmacological management should be used sparingly for BPSD only after non-pharmacological approaches have failed (Ngo and Holroyd-Leduc, 2014). Overuse of antipsychotics in order to control the BPSD leads to high cost and harmful side effects, emphasizing the benefit of a combination approach of pharmacological and non-pharmacological treatments (Haight et al., 2006; van Bogaert et al., 2013). Vascular dementia $(\mathrm{VaD})$ patients, especially those with small vessel disease, present with social inactivity or apathy as a one of the major problems (Staekenborg et al., 2010); thus, psychosocial interventions and drug treatments should be encouraged. These results are consistent with those of our meta-analysis.

Our result showed that reminiscence therapy had a small to medium sized effect on depression, QoL, and BPSD in PWDs. Studies including in this current meta-analysis had various sample sizes, outcomes, countries of subject origin, and even different effect sizes. Many researchers and staff in long-term care settings have applied reminiscence therapy as a non-pharmacological intervention, regardless of its effectiveness in PWDs. One of the recent dementia practice guidelines (Ngo and Holroyd-Leduc, 2014) recommended that pharmacological intervention to manage the BPSD should be used only after nonpharmacological intervention has failed. This guideline also recommended that non-pharmacological intervention such as reminiscence therapy be initially applied to manage mood symptoms. We expect that this guideline and the findings from this current study will encourage staffs in long-term care settings to develop and apply reminiscence therapy as a clinically appropriate method to decrease depression and BPSD and QoL for PWDs.

With regard to the result of the subgroup analysis for group versus individual reminiscence therapy, the overall mean effect size of the depression variable was -0.785 (95\% CI: -1.191 to $-0.378, \mathrm{Z}=$ $-5.451, p<0.001)$ from 5 RCTs that conducted individual reminiscence therapy, and $-0.466(95 \%$ CI: -0.798 to $-0.135, Z=-2.759, p=0.006)$ from 12 RCTs that conducted group reminiscence therapy. Therefore, we suggested that the individual approach had a stronger benefit to reduce depression than the group approach for PWDs, specifically the patient-focused intervention and patient centered interview (Hilgeman et al., 2014; Woods et al. , 2018). Moreover, the life review process through reminiscence therapy needs to be developed as an individual approach, because the individual approach was relevant for individuals who wished to discuss one life stage for longer than anticipated (Morgan and Woods, 2012). In another subgroup analysis for length of study time, the overall mean effect sizes of the depression, QoL, and BPSD in reminiscence group with less than 8 sessions were significantly different, but the overall mean effect sizes of the QoL and BPSD in reminiscence group with less than 6 sessions or 4 sessions were not significantly different. Therefore, we suggested that reminiscence therapy of more than 8 sessions might be required to obtain the therapeutic effects on the QoL and BPSD.

There were some limitations in this study. First, we did not separately analyze the effects of group and individual reminiscence therapy. Second, the study setting was not distinguished, so some of the treatments occurred in care homes, while others were in community settings. Third, in this study, the study participants were not able to distinguish by neither the types of dementia nor diagnosis of dementia, therefore our results could not describe the differences in effects by reminiscence therapy; however, because Tadaka and Kanagawa (2007) found differences in MMSE scores between vascular dementia and Alzheimer's dementia, future study is suggested to examine the effects of reminiscence therapy on cognition as measured by MMSE according to the types of dementia.

\section{Conclusion}

This systematic review and meta-analysis provide the evidence that reminiscence therapy can have beneficial effects on depression, QoL, and BPSD in PWD. A heterogeneity test of effect size under a random effects model was performed. Depression and BPSD were significantly decreased, and QoL were significantly increased in the reminiscence group compared to the control group. Therefore, reminiscence therapy could be broadly used to control depression as an alternative to antipsychotics with harmful side effects or high cost.

\section{Conflict of interest}

There are no conflicts of interest to disclose.

\section{Description of author's roles}

GR. Hong and K. Park designed the study, K. Park and $S$. Lee reviewed the selected articles and rated the risks of bias, J. Yang and T. Song reviewed the literature and organized them. K. Park was 
responsible for carrying out the statistical analysis. All authors drafted the article and critically reviewed it. All contributing authors have read and approved the final version of the manuscript.

\section{Acknowledgments}

This work was supported by the National Research Foundation of Korea (NRF) grant funded by the Korean government (Ministry of Science, ICT, \& Future Planning: MSIP) (NO. NRF-2013R1A1A2 073275).

\section{References}

Akanuma, K. et al. (2011). Improved social interaction and increased anterior cingulate metabolism after group reminiscence with reality orientation approach for vascular dementia. Psychiatry Research, 192, 183-187. doi: 10.1016/j .pscychresns.2010.11.012.

American Psychiatric Association (2007). Practice Guideline for the Treatment of Patients with Alzheimer's Disease and Other Dementias. Arlington: American Psychiatric Association.

Azcurra, D. J. L. S. (2012). A reminiscence program intervention to improve the quality of life of long-term care residents with Alzheimer's disease. A randomized controlled trial. Revista Brasileira de Psiquiatria, 34, 422-433. doi: 10.1016/j.rbp.2012.05.008.

Bailar, J. C. (1997). The promise and problems of meta-analysis. The New England fournal of Medicine, 337, 559-561. doi: 10.1056/nejm199708213370810.

Baines, S., Saxby, P. and Ehlert, K. (1987). Reality orientation and reminiscence therapy. A controlled cross-over study of elderly confused people. The British Fournal of Psychiatry, 51, 222-231. doi: 10.1192/bjp. 151.2.222.

Begg, C. B. and Mazumdar, M. (1994). Operating characteristics of a rank correlation test for publication bias. Biometrics, 50, 1088-1101.

Buchanan, K. and Middleton, D. (1994). Reminiscence reviewed: a discourse analytic perspective. In J. Bornat (Ed.), Reminiscence Reviewed: Perspectives, Evaluations, Achievements (pp. 61-73). Buckingham, UK: Open University Press.

Butler, R. N. (1963). The life review: an interpretation of reminiscence in the aged. Psychiatry, 26, 65-76.

Carrion, C., Aymerich, M., Baillés, E. and LópezBermejo, A. (2013). Cognitive psychosocial intervention in dementia: a systematic review. Dementia and Geriatric Cognitive Disorders, 36, 363-375. doi: 10.1159/000354365.

Chalmers, T. C. (1991). Problems induced by metaanalyses. Statistics in Medicine, 10, 971-980. doi: 10.1002/ sim.4780100618.

Chang, W. S. and Je, S. (2008). A comparative study on the effect of group reminiscence therapy and group validation therapy for the elderly with dementia. Fournal of Welfare for the Aged, 41, 301-325.
Chang, W. S. and Lee, J. M. (2006). The effect of group reminiscence therapy on depression, quality of life and social behavior of patient with dementia. Fournal of Welfare for the Aged, 34, 239-269.

Chin, A. M. H. (2007). Clinical effects of reminiscence therapy in older adults: a meta-analysis of controlled trials. Hong Kong Fournal of Occupational Therapy, 17, 10-22. doi: 10.1016/S1569-1861(07)70003-7.

Committee for Proprietary Medicinal Products (2000). Points to Consider on Switching between Superiority and Non-Inferiority. London: The European Agency for the Evaluation of Medicinal Products.

Duru Aşiret, G. and Kapucu, S. (2016). The effect of reminiscence therapy on cognition, depression, and activities of daily living for patients with Alzheimer disease. Fournal of Geriatric Psychiatry and Neurology, 29, 31-37. doi: 10.1177/0891988715598233.

Felson, D. T. (1992). Bias in meta-analytic research. fournal of Clinical Epidemiology, 45, 885-892. doi: 10.1016/08954356(92)90072-U.

Gitlin, L. N. et al. (2009). The tailored activity program to reduce behavioral symptoms in individuals with dementia: feasibility, acceptability, and replication potential. The Gerontologist, 49, 428-439. doi: 10.1093/geront/gnp087.

Goldwasser, A. N., Auerbach, S. M. and Harkins, S. W. (1987). Cognitive, affective, and behavioral effects of reminiscence group therapy on demented elderly. The International fournal of Aging and Human Development, 25, 209-222.

Gottfries, C. G. (2001). Late life depression. European Archives of Psychiatry and Clinical Neuroscience, 251, 57-61. doi: 10.1007/BF03035129.

Gupta, S. K. (2011). Intention-to-treat concept: a review. Perspectives in Clinical Research, 2, 109-112. doi: 10.4103/ 2229-3485.83221.

Haight, B. K., Gibson, F. and Michel, Y. (2006). The Northern Ireland life review/life storybook project for people with dementia. Alzheimers $\mathcal{E}$ Dementia, 2, 56-58. doi: 10.1016/j.jalz.2005.12.003.

Hartling, L. et al. (2012). Validity and Inter-Rater Reliability Testing of Quality Assessment Instruments. Rockville, MD: Agency for Healthcare Research and Quality (US).

Higgins, J. P. T. and Altman, D. G. (2008). Assessing risk of bias in included studies. In J. P. T. Higgins and S. Green (Eds.), Cochrane handbook for systematic reviews of interventions (pp. 187-241). Chichester: John Wiley \& Sons, Ltd.

Higgins, J. P. T., Thompson, S. G., Deeks, J. J. and Altman, D. G. (2003). Measuring inconsistency in meta-analyses. BMF, 327, 557. doi: $10.1136 / \mathrm{bmj} .327$ .7414 .557 .

Higgins, J. P. T. et al. (2011). The Cochrane Collaboration's tool for assessing risk of bias in randomised trials. BMF, 343, d5928. doi: 10.1136/bmj.d5928.

Hilgeman, M. M., Allen, R. S., Snow, A. L., Durkin, D. W., DeCoster, J. and Burgio, L. (2014). Preserving Identity and Planning for Advance Care (PIPAC): preliminary outcomes from a patient-centered intervention for individuals with mild dementia. Aging $\mathcal{E}$ Mental Health, 18, 411-424. doi: 10.1080/13607863.2013 .868403 . 
Hollis, S. and Campbell, F. (1999). What is meant by intention to treat analysis? Survey of published randomised controlled trials. BMF, 319, 670-674. doi: 10.1136/bmj .319.7211.670.

Hsieh, C. J. et al. (2010). Reminiscence group therapy on depression and apathy in nursing home residents with mild-to-moderate dementia. Fournal of Experimental $\mathcal{E}$ Clinical Medicine, 2, 72-78.

Huang, H. C. et al. (2015). Reminiscence Therapy improves cognitive functions and reduces depressive symptoms in elderly people with dementia: a meta-analysis of randomized controlled trials. $\mathcal{F} A M D A, 16,1087-1094$. doi: 10.1016/j.jamda.2015.07.010.

Ito, T., Meguro, K., Akanuma, K., Ishii, H. and Mori, E. (2007). A randomized controlled trial of the group reminiscence approach in patients with vascular dementia. Dementia Geriatric Cognitive Disorders, 24, 48-54. doi: 10.1159/000103631.

Kwon, M., Jung, D. and Kim, S. (2012). The effects of group reminiscence program on social interaction and behavioral problems in the resident elderly with dementia. The Fournal of Occupational Therapy for the Aged and Dementia, 6, 11-18.

Lai, C. K. Y., Chi, I. and Kayser-Jones, J. (2004). A randomized controlled trial of a specific reminiscence approach to promote the well-being of nursing home residents with dementia. International Psychogeriatrics, 16, 33-49. doi: 10.1017/s1041610204000055.

Moher, D., Schulz, K. F. and Altman, D. G. (2001). The CONSORT statement: revised recommendations for improving the quality of reports of parallel group randomized trials. BMC Medical Research Methodology, 1, 2-7. doi: 10.1186/1471-2288-1-2.

Morgan, S. and Woods, R. T. (2012). Life review with people with dementia in care homes: a preliminary randomized controlled trial. Non-Pharmacological Therapies in Dementia, 1, 43-59.

Nakamae, T., Yotsumoto, K., Tatsumi, E. and Hashimoto, T. (2014). Effects of productive activities with reminiscence in occupational therapy for people with dementia: a pilot randomized controlled study. Hong Kong fournal of Occupational Therapy, 24, 13-19. doi: 10.1016/j .hkjot.2014.01.003.

Nakatsuka, M. et al. (2015). A cluster randomized controlled trial of nonpharmacological interventions for oldold subjects with a Clinical Dementia Rating of 0.5: the Kurihara Project. Dementia and Geriatric Cognitive Disorders Extra, 5, 221-232. doi: 10.1159/000380816.

Ngo, J. and Holroyd-Leduc, J. M. (2014). Systematic review of recent dementia practice guidelines. Age and Ageing, 44, 25-33.

O'Shea, E. et al. (2014). The impact of reminiscence on the quality of life of residents with dementia in long-stay care. International fournal of Geriatric Psychiatry, 29, 1062-1070. doi: 10.1002/gps.4099.

Staekenborg, S. S. et al. (2010). Behavioural and psychological symptoms in vascular dementia; differences between small- and large-vessel disease. Fournal of
Neurology, Neurosurgery $\mathcal{E}$ Psychiatry, 81, 547-551. doi: 10.1136/jnnp.2009.187500.

Stinson, C. K. (2009). Structured group reminiscence: an intervention for older adults. The fournal of Continuing Education in Nursing, 40, 521-528.

Subramaniam, P. and Woods, B. (2012). The impact of individual reminiscence therapy for people with dementia: systematic review. Expert Review of Neurotherapeutics, 12, 545-555.

Subramaniam, P., Woods, B. and Whitaker, C. (2014). Life review and life story books for people with mild to moderate dementia: a randomised controlled trial. Aging E Mental Health, 18, 363-375. doi: 10.1080/13607863 .2013 .837144

Tadaka, E. and Kanagawa, K. (2007). Effects of reminiscence group in elderly people with Alzheimer disease and vascular dementia in a community setting. Geriatrics $\mathcal{E}$ Gerontology International, 7, 167-173. doi: 10.1111/j .1447-0594.2007.00381.x.

Thorgrimsen, L., Schweitzer, P. and Orrell, M. (2002). Evaluating reminiscence for people with dementia: a pilot study. The Arts in Psychotherapy, 29, 93-97.

van Bogaert, P., Van Grinsven, R., Tolson, D., Wouters, K., Engelborghs, S. and Van der Mussele, S. (2013). Effects of SolCos model-based individual reminiscence on older adults with mild to moderate dementia due to Alzheimer disease: a pilot study. Fournal of the American Medical Directors Association, 14, 528-e9. doi: 10.1016/j.jamda.2013.01.020.

Wang, J. J. (2007). Group reminiscence therapy for cognitive and affective function of demented elderly in Taiwan. International fournal of Geriatric Psychiatry, 22, 1235-1240. doi: 10.1002/gps.1821.

Wang, J. J., Yen, M. and OuYang, W. C. (2009). Group reminiscence intervention in Taiwanese elders with dementia. Archives of Gerontology and Geriatrics, 49, 227-232. doi: 10.1016/j.archger.2008 .08 .007 .

Wertz, R. T. (1995). Intention to treat: once randomized, always analyzed. Clinical Aphasiology, 23, 57-64.

Woods, B., O'Philbin, L., Farrell, E. M., Spector, A. E. and Orrell, M. (2018). Reminiscence therapy for dementia. Cochrane Database of Systematic Reviews, 3, CD001120. doi: 10.1002/14651858 .CD001120.pub3.

Woods, B., Spector, A., Jones, C., Orrell, M. and Davies, S. (2005). Reminiscence therapy for dementia. Cochrane Database Systematic Reviews, 2, CD001120.

Woods, R., Bruce, E., Edwards, R., Elvish, R. and Hoare, Z. (2012). REMCARE: reminiscence groups for people with dementia and their family caregivers effectiveness and cost-effectiveness pragmatic multicentre randomised trial. Health Technology Assessment, 16, 1-121. doi: $10.3310 /$ hta16480.

World Health Organization and Alzheimer's Disease International (2012). Dementia: A Public Health Priority. Geneva: World Health Organization. 


\section{Appendix: List of Abbreviations}

$\begin{array}{ll}\mathrm{AD} & =\text { Alzheimer's Disease } \\ \mathrm{ADL} & =\text { Activities of Daily Living } \\ \mathrm{ADQ} & =\text { Approaches to Dementia Questionnaire } \\ \mathrm{AES} & =\text { Apathy Evaluation Scale } \\ \mathrm{AIS} & =\text { Autobiographical Incidents Schedule } \\ \mathrm{AMI} & =\text { Autobiographical Memory Interview } \\ \mathrm{AMS} & =\text { Alzheimer Mood Scale } \\ \mathrm{BRS} & =\text { Behavioral Rating Scale } \\ \mathrm{BRSE} & =\text { Behavioral Rating Scale for the Elderly } \\ \mathrm{CAPE} & =\text { Clifton Assessment Procedures for the } \\ & \text { Elderly } \\ \mathrm{CAS} & =\text { Cognitive Assessment Scale } \\ \mathrm{CMAI} & =\text { Cohen-Mansfield Agitation Inventory } \\ \mathrm{COS} & =\text { Communication Observation Scale } \\ \mathrm{CPS} & =\text { Cognitive Performance Scale } \\ \mathrm{CSDD} & =\text { Cornell Scale for Depression in Dementia } \\ \mathrm{DCS} & =\text { Depressive Cognition Scale } \\ \mathrm{EQ}-5 \mathrm{DD} & =\text { Euro Quality Of Life -5Dimensions } \\ \text { FAB } & =\text { Frontal Assessment Battery } \\ \text { FIM } & =\text { Functional Independence Measure } \\ \text { GDS } & =\text { Geriatric Depression Scale } \\ \text { GDS-SF } & =\text { Geriatric Depression Scale-Short Form } \\ \text { GHQ } & =\text { General Health Questionnaire } \\ \text { GQoL-D } & =\text { Geriatric Quality Of Life Dementia }\end{array}$

IMMEL = Index for Managing Memory Loss

NPI $=$ Neuropsychiatric Inventory

MBP = Memory Behavior Problem

MBPC $=$ Memory \& Behavior Problem Checklist

MDS- $\quad=$ Minimal Data Set Activities of Daily

ADL Living

MMSE $=$ Mini-Mental State Examination

MOSES $=$ Multidimensional Observation Scale for Elderly Subjects

MZBI = Modified Zarit Burden Interview

PSS $\quad=$ Perceived Semantic Scale

QCPR = Quality of the Caregiving Relationship

QoL $\quad=$ Quality Of Life

QoL-AD = Quality of life in Alzheimer's Disease

RAID $\quad=$ Rating Anxiety in Dementia

RT $\quad=$ Reminiscence Therapy

RSS $\quad=$ Relatives Stress Scale

SBS $\quad=$ Social Behavior Scale

SES = Social Engagement Scale

SRQoL = Self Reported Quality Of Life

TMT-A $=$ Trail Making Test part A

UK $=$ United Kingdom

USA $\quad=$ United States of America

VD $\quad=$ Vascular Dementia

WF $\quad=$ Word Fluency

WIB $\quad=$ Well/Ill -Being

ZBI $=$ Zarit Burden Interview 